\title{
Characterization of conjunctival microbiome dysbiosis associated with allergic conjunctivitis
}

\author{
Qiaoxing Liang ${ }^{1}$, Jing $\mathrm{Li}^{1}$, Shiyao Zhang ${ }^{1}$, Yinglin Liao ${ }^{1}$, Shixin Guo ${ }^{1}$, Juanran Liang ${ }^{1}$, \\ Xiuli Deng ${ }^{1}$, Yu Liu ${ }^{1}$, Bin Zou ${ }^{1}$, Xiaofeng Wen ${ }^{1}$, Lingyi Liang ${ }^{1}$, and Lai Wei ${ }^{1}$ \\ ${ }^{1}$ Sun Yat-Sen University Zhongshan Ophthalmic Center
}

August 11, 2020

\begin{abstract}
Background: Evidence shows that the microbiome plays an important role in allergic diseases. The associations between the conjunctival microbiome dysbiosis and ocular allergy remain unknown. This study investigates the characteristics of the conjunctival microbiome in allergic conjunctivitis (AC). Methods: We performed metagenomic shotgun sequencing on conjunctival samples from 48 healthy subjects and 39 patients with AC. The AC group comprised 21 patients with seasonal allergic conjunctivitis or perennial allergic conjunctivitis (SAC/PAC) and 18 patients with vernal keratoconjunctivitis (VKC). Results: We identified a signature microbiome associated with the dysbiosis of conjunctival microbiota on the ocular surface of patients with AC. Interestingly, SAC/PAC and VKC harbor distinct microbial communities. Specifically, Brevibacterium aurantiacum is enriched in SAC/PAC, whereas a group of Streptococcus species and Haemophilus parainfluenzae are enriched in VKC. Conclusion: Dysbiosis of conjunctival microbiome is found on the ocular surface of AC patients. These results suggest a new microbial mechanism of $\mathrm{AC}$ and provide a potential target for $\mathrm{AC}$ treatment.
\end{abstract}

\section{INTRODUCTION}

Allergic diseases have dramatically risen over the last few decades. ${ }^{1}$ Allergic conjunctivitis (AC) is one of the most common ocular surface disorders that encompasses a spectrum of diseases featured by antigen-specific immunoglobulin $\mathrm{E}$ (IgE) and $\mathrm{T}$ helper type 2 (Th2) lymphocyte-mediated hypersensitivity responses. ${ }^{2}$ Among different types of AC, seasonal allergic conjunctivitis (SAC) and perennial allergic conjunctivitis (PAC) represent the majority of cases. SAC and PAC share similar clinical feature and pathologic process in which type I hypersensitivity plays a key role. Vernal keratoconjunctivitis (VKC) and atopic keratoconjunctivitis (AKC) are more severe than $\mathrm{SAC} / \mathrm{PAC}$ and can ultimately lead to vision loss. In contrast to $\mathrm{SAC} / \mathrm{PAC}$, type IV hypersensitivity is the major mechanism of VKC/AKC.

Increasing evidence shows that the microbiome of skin, gut, and airways play an important role in allergic diseases like atopic dermatitis, food allergies, and asthma. ${ }^{3,4}$ For instance,Staphylococcus aureus exacerbates atopic dermatitis by inducing skin inflammation and weakening the skin barrier. ${ }^{5}$ Colonization of Moraxella , Streptococcus, andHaemophilus in the airways are associated with increased risk of asthma possibly by promoting viral respiratory tract infections. ${ }^{6}$ Similar to skin and other mucosal surfaces, conjunctiva is colonized with a variety of microbes. ${ }^{7-10}$ However, the association between the conjunctival microbiome dysbiosis and ocular allergy remains unexplored.

Here we surveyed the conjunctival metagenomes of 39 patients with various types of AC (SAC/PAC and VKC) and 48 healthy controls using metagenomic shotgun sequencing. We characterized the microbial dysbiosis associated with $\mathrm{AC}$ and identified distinct microbial signatures of $\mathrm{VKC}$ and $\mathrm{SAC} / \mathrm{PAC}$. These findings offer new insight into the prevention and treatment of ocular allergy.

\section{METHODS}




\subsection{Participant recruitment}

The study was conducted in accordance with the tenets of the Declaration of Helsinki and was approved by the Ethics Committee of Zhongshan Ophthalmic Center, Sun Yat-sen University (Guangzhou, China). Written informed consent was obtained from all participants after explanation of the nature and possible consequences of the study.

Participants with an established AC diagnosis were recruited at Zhongshan Ophthalmic Centre from December 2017 through June 2018. Both eyes of the participants were tested for all clinical parameters. The inclusion criteria and classification of AC were based on the Japanese guidelines for allergic conjunctival diseases 2017. ${ }^{11}$ Potential participants were excluded if they received topical anti-bacterial treatment in a recent year; systemic antibiotics within 90 days; topical or systemic corticosteroids within 1 week; topical CsA, tacrolimus, or any systemic immunosuppressive drug within 90 days; scrape on the vernal plaque within 1 month; any other ocular surgery within 6 months; or if they presented or had a history of severe systemic allergy at the study entry. Our published metagenomic data of the 48 healthy young subjects were used as healthy controls in this study. ${ }^{10}$

\subsection{Sample collection}

Both eyes of the patients diagnosed with $\mathrm{AC}$ were screened, and the eye with severer symptoms was sampled for sequencing. The conjunctival impression samples were collected and stored using a previously described protocol. ${ }^{10,12}$ Briefly, a sterile semicircle MF membrane filter (REF: HAWP01300, $0.45 \mathrm{~lm}$ in diameter; Merck Millipore, Burlington, MA, USA) was placed on the inferior bulbar conjunctiva for 10 seconds. The membrane was then immediately placed in a sterile tube with $300 \mu \mathrm{l}$ of Tissue and Cell Lysis Solution (Epicentre, Ambleside, UK) and stored at -80.

\subsection{DNA isolation and metagenomic shotgun sequencing}

The DNA isolation and metagenomic shotgun sequencing of the AC samples was performed as previously described. ${ }^{10,12}$ In brief, DNA was extracted from each conjunctival sample using the MasterPure Complete DNA and RNA Purification Kit (Epicentre) according to the manufacturer's instructions. Sequencing libraries were prepared using the VAHTS Universal DNA library Prep Kit for Illumina (Vazyme, Nanjing, China) and quantified by qPCR using the KAPA SYBR FAST qPCR Kit (Kapa Biosystems, Wilmington, MA, USA). Paired-end $2 \times 150$-bp sequencing was performed on a NovaSeq 6000 instrument (Illumina, San Diego, CA, USA). Three negative blank controls, which had reagents from DNA extraction through sequencing, were processed along with the AC samples.

\subsection{Taxonomic and functional profiling}

The sequencing reads were first quality filtered using Trimmomatic ${ }^{13}$ v0.36 and PRINSEQ ${ }^{14}$ v0.20.4. Human reads were removed using KneadData v0.6.1 (https://bitbucket.org/biobakery/kneaddata). High quality nonhuman reads were mapped against a custom database using Kraken $2^{15}$ v2.0.9. A total of 29,943 complete microbial genomes were downloaded on 3 May 2020, of which 19,362 were bacterial, 368 were archaeal, 9,346 were viral, and 867 were fungal. The complete bacterial, archaeal, and viral genomes were downloaded from RefSeq database using the -download-library option of kraken2-build. The complete fungal genomes were manually downloaded from GenBank database. The results of taxonomic classification were filtered using a confidence score of 0.20 . Only species with more than 10 reads in at least one sample were retained. The species profiles were decontaminated (see below) and the reads derived from non-contaminant species were used for functional analysis. Functional profiling was performed using HUMAnN2 ${ }^{16}$ v0.11.1. The abundance profiles of gene families (UniRef90s) were summarized to the abundance of KEGG orthology (KO), Enzyme Commission (EC) gene families, EggNOG clusters of orthologous groups, and Pfam protein families, respectively. In addition, the non-contaminant reads were mapped against the protein homolog sequences of the antimicrobial resistance genes in CARD database ${ }^{17}$ (May 2020 release) using DIAMOND ${ }^{18}$ v0.9.22.123.

\subsection{Decontamination pipeline}


Contaminant species were detected and removed using the decontam $\mathrm{R}$ package. ${ }^{19}$ We used the most stringent hyperparameter value $\left(P^{*}=0.5\right)$ for both frequency-based and prevalence-based contaminant identification of the is Contaminant function. DNA concentrations for the frequency method were measured by qPCR and were obtained during library preparation. The scores from the frequency and prevalence methods were combined using the "minimum" approach. We performed decontamination in batches and the species identified as contaminants in any batch were removed. The species not identified as contaminants with decontam were further excluded if they met one of the following criteria: (1) The relative abundance of a species show an inverse correlation with DNA concentration ${ }^{20}(\rho<-0.2, P<0.05$, Spearman's correlation); (2) The relative abundance of a species $>0.01 \%$ in at least one negative blank control.

\subsection{Statistical analyses}

Statistical analyses were performed using R (v3.5.1) software packages. Shannon diversity indices and BrayCurtis dissimilarity indices were respectively computed by the functions diversity andvegdist in the vegan $\mathrm{R}$ package. Two-sided Wilcoxon's rank-sum test was used for comparisons of Shannon diversity and Bray-Curtis dissimilarity indices between two groups. Two-sided Fisher's exact test was used to compare the occurrence of antibiotic resistance genes. Principal coordinates analysis $(\mathrm{PCoA})$ was performed using thedudi.pco function in the ade4 $\mathrm{R}$ package. The species and gene family signatures were identified using LEfSe. ${ }^{21}$ Briefly, KruskalWallis rank-sum test was used to detect features with significantly differential abundance between two groups. LEfSe then applied linear discriminant analysis (LDA) on these features to estimate their effect sizes in delineation between different disease states. For all boxplots, the box edges denoted the first and third quartiles and the horizontal line denoted the median, with the whiskers extending up to the 1.5 -fold interquartile ranges.

\section{RESULTS}

\subsection{Dysbiosis of conjunctival microbiome in allergic conjunctivitis}

The study cohort was composed of 39 individuals with AC and 48 healthy subjects (Table S1). The group of AC comprised 14 patients with PAC, 7 with SAC, and 18 with VKC (Table S2). Table S1 and Table S2 show the age at sample collection. All the patients were in active stage of AC with inflamed ocular surface. We performed shotgun metagenomic sequencing and obtained on average 0.32 million high quality non-human reads for each individual. A total of 278 species passed our decontamination pipeline (Methods).

Overall, bacteria accounted for the majority of the conjunctival microbiota of both healthy and AC individuals (Figure 1A). We observed the enrichment of eukaryotic virus in a subset of healthy individuals (KruskalWallis rank-sum test $P=0.0014$ ). The prominent viral species in these samples was human beta herpesvirus 7 , which is frequently detected and rarely pathogenic in immunocompetent individuals. ${ }^{22}$ Malassezia Fungi (M.furfur in particular) were abundant in a fraction of patients with SAC/PAC (but not VKC) compared with healthy individuals (Figure 1B; $P=0.0095$ ). IgE mediated hypersensitivity to Malasseziaspecies correlates with the clinical severity of atopic dermatitis (AD) ${ }^{23}$ Malassezia produces immunogenic proteins that elicit IgE and thus induce pro-inflammatory cytokines and auto-reactive $\mathrm{T}$ cells, which contributes to $\mathrm{AD}$ pathogenesis. ${ }^{24}$ Notably, SAC/PAC is dominated by IgE-mediated reactions in contrast to VKC.

The alpha diversity showed no significant difference between healthy and AC individuals (Figure 2A), whereas the Bray-Curtis dissimilarities within AC groups were slightly lower than healthy groups (Figure 2B). The principal coordinates analysis $(\mathrm{PCoA})$ of the species composition showed a clear delineation between healthy and $\mathrm{AC}$ participants (Figure $2 \mathrm{C}$ ), suggesting that dysbiosis of the conjunctival microbiome is associated with AC.

To identify the species that accounts for the dysbiosis, we performed LEfSe analysis on the species profiles and the species with LDA effect size $>4$ were displayed in Figure 2D. Numerous species were overabundant on the ocular surface of AC patients compared to healthy subjects (Figure 2E), such as members of Oxalobacteraceae including Janthinobacterium $s p$. Marseille $\left(P=1.6 \times 10^{-17}\right)$, Herminiimonas arsenitoxidans $\left(P=2.3 \times 10^{-14}\right)$, and Herminiimonasarsenicoxydans $\left(P=5.2 \times 10^{-15}\right)$. Interestingly, family Oxalobacteraceae was prevalently 
detected in the conjunctival microbiota of lens wearers ${ }^{25}$ and contact lens wearing is in turn associated with ocular allergy. ${ }^{26}$ Rothia was also enriched in samples from participants with AC, including Rothia aeria $\left(P=1.8 \times 10^{-4}\right)$ and Rothia dentocariosa $(P=0.0010)$. In addition, we detected the enrichment of Moraxella catarrhalis in AC $(P=0.0033)$. Moraxella catarrhalis in the upper airways is linked with the development or exacerbation of allergic airway inflammation and IL-17 and TNF- $\alpha$ are involved in this process. ${ }^{27}$ Colonization of $M$. catarrhalis in the airways leads to a low-grade systemic inflammation that is associated with established asthma. ${ }^{28}$ This implies that common microbial mechanisms may underlie both ocular allergy and allergic diseases prevalent at other sites.

We next searched for functional differences in the conjunctival microbiome between healthy and AC individuals. Most of the gene families identified using LEfSe were enriched in the metagenome from the AC patients (Figure 3A). For instance, we detected the enrichment of the gene families related to amino acid metabolism such as aspartate kinase (Kruskal-Wallis rank-sum test $P=0.0058)$, tryptophan synthase $(P$ $=0.012)$, and glutamine synthetase $(P=0.026)$. In addition, the gene families encoding thioredoxin $(P$ $=0.012)$ and thioredoxin reductase $(P=0.026)$ were more abundant in AC patients compared to healthy individuals.

Exposure to antibiotics has been associated with many allergic diseases. ${ }^{29}$ Therefore, we further examined the prevalence of antibiotic resistance genes in the conjunctival metagenome of AC patients (Figure 3B). In particular, we observed that the genes resistant to tetracycline is more prevalent in AC patients than healthy individuals, including tet $A$ (Fisher's exact test $P=0.045), \operatorname{tet} B(P=0.0043), \operatorname{optr} A(P=0.038), \operatorname{vmlR}$ $(P=0.0026)$, and $\operatorname{evg} S(P=0.042)$. Glycopeptide antibiotic resistance genes such as $\operatorname{vanTG}(P=0.016)$ and $\operatorname{van} G(P=0.037)$ were detected exclusively in $\mathrm{AC}$ samples. Fluoroquinolone antibiotic resistance genes were also more prevalent in the samples from AC patients compared to healthy subjects, including acrF ( $P$ $=0.037), \operatorname{efr} B(P=0.031), \operatorname{evg} S(P=0.042), \operatorname{lfr} A(P=0.037)$, and patA $(P=0.038)$. This warrants further studies into the relationships between antibiotic use and the risk of ocular allergy.

\subsection{Distinct microbial signatures of $\mathrm{SAC} / \mathrm{PAC}$ and $\mathrm{VKC}$}

Apart from the differences in manifestation and incidence, SAC/PAC and VKC are driven by different immune reactions. Previous studies suggested VKC are mainly related to Th2-type allergic inflammation while SAC/PAC manifest the classical type I hypersensitivity. ${ }^{30}$ In addition, type IV hypersensitivity is involved in VKC. We thus asked whether the conjunctival microbiota was different between SAC/PAC and VKC. Although the alpha diversity of the entire AC group showed no difference to the healthy group, the conjunctival microbiota of the patients with $\mathrm{SAC} / \mathrm{PAC}$ was less diverse than healthy subjects (Figure $4 \mathrm{~A} ; P$ $=0.043)$. We did not find significant difference in inter-individual variation within samples of SAC/PAC and VKC (Figure 4B; $P=0.31$ ). As expected, the species composition of conjunctival microbiome distinguished between SAC/PAC and VKC (Figure 4C). We then performed linear discriminant analysis to identify the species signatures of SAC/PAC and VKC (Figure 4D).

The distribution of species that showed differences in abundance between SAC/PAC and VKC were summarized in Figure 5A. Brevibacterium aurantiacum was enriched in SAC/PAC (Figure 5B; Kruskal-Wallis rank-sum test $P=0.0062$ ). We observed the enrichment ofStreptococcus species in VKC (Figure 5C), includingStreptococcus pneumoniae $(P=0.033)$, Streptococcus salivarius $(P=0.022)$, and Streptococcus sanguinis $(P=0.034)$. Haemophilus parainfluenzae $(P=0.041)$, Actinomyces $s p$. oral taxon $848(P=$ $0.026)$, and Campylobacter concisus $(P=0.013)$ were also more abundant and more prevalent in VKC compared with SAC/PAC. Colonization ofS. pneumoniae and Haemophilus Influenzae in airways are also the risk factors of asthma. ${ }^{28}$ Campylobacter in lower airways is associated with resistance to corticosteroids in asthma. ${ }^{31}$ Interesting, alpha-hemolytic Streptococcus (which includes S. pneumoniae and S. sanguinis ) and H. Influenzae were observed on the ocular surface of oGVHD patients using culture-based methods. ${ }^{32}$ These results indicate that the composition of conjunctival microbiota is associated with specific types of AC, which potentially provides novel aspects for the prevention and treatment of ocular allergy.

\section{DISCUSSION}


Ocular allergy represents one of the most common ocular surface diseases. Despite an emerging picture of immunologic mechanisms underlying AC, the etiology is not fully understood especially for severe forms like VKC. In this study, we characterized the associations of conjunctival microbiome dysbiosis with AC and identified the species involved in the dysbiosis. Though it is unclear which aspects of the microbial dysbiosis are causes or consequences of the onset of diseases, these findings captured novel characteristics of AC. Future studies are needed to clarify the roles of the discovered associated species in the development and exacerbation of ocular allergy. Current treatment regimens of AC mainly focus on suppressing inflammation, and combining these first-line treatments with therapies targeting the ocular surface microbiome may be beneficial.

The age distribution of the AC participants was older than epidemiologically expected. This might be owing to temporal interval between the first onset of $\mathrm{AC}$ and the time when they came to our hospital. Although the age of healthy and AC participants were not exactly matched, the age-associated species were absent from the species identified in this study. ${ }^{10}$ This implies that age is not the driver of microbiome differences between healthy and $\mathrm{AC}$ participants in our study. In addition, it is unknown if the colonization of these species predates the onset of AC (especially in early life), highlighting the need for longitudinal studies to investigate the transition to microbial dysbiosis in AC.

We did not find significant differences in species composition between SAC and PAC, which is consistent with their similarity in signs and symptoms. Most of the species with different abundance between SAC/PAC and VKC were enriched in VKC. The involvement of type IV hypersensitivity might be related to the overgrowth of these species. In clinical practice, the allergens of SAC and PAC are often clear, whereas the triggers of VKC are generally difficult to find. This highlights the value of investigating the roles of microorganisms in VKC.

The incidence of allergic diseases is influenced by multiple factors. For instance, personal life experiences lead to distinct allergen profiles across individuals. Despite enrichment in AC, some species were also present in healthy individuals, suggesting that other factors might contribute to the pathogenicity of these species. Moreover, some individuals with $\mathrm{AC}$ harbored one or a few prominent species unlike other patients. For example, a sample from an eight-year-old boy with VKC contained a non-negligible abundance $(>10 \%)$ of human beta herpesvirus 6A. This reflects the complexity and individuality of microbial mechanisms in AC.

It is of particular interest that some species enriched in AC have been previously shown to contribute to other allergic diseases like atopic dermatitis and asthma. This suggests that various allergic disorders might have some microbial mechanisms in common. Specifically, the ocular surface is connected with the nasopharynx through the nasolacrimal canal. This connection may facilitate the exchange of pathogenic species. Further studies are required to provide evidence for the similar roles of these species on conjunctiva as on other mucosal surfaces. Apart from local airways microbiome, gut microbiome also plays a part in asthma. Likewise, the association of gut microbiome and AC is worth investigating.

Of note, many of the species involved in AC overlapped the most niche-associated (showing great phylogenetic separation between body sites) species identified by the Human Microbiome Project, ${ }^{33}$ such as Haemophilus parainfluenzae, Rothia dentocariosa, and Streptococcus sanguinis. It is very likely that ocular surface harbors unique strains of these species emphasizing the need for further efforts to characterize the genomes of species living on the ocular surface. These efforts may include whole genome sequencing on the isolates cultured from ocular surface samples, as well as large-scaled metagenome assembly. Further investigation will lead to a better understanding of how specific strains of these microorganisms are associated with AC.

Besides small sample size, a limitation of this study is that AKC was not included. AKC is least common and its pathologic mechanism is considered similar with that of VKC. In addition, we only sampled from inferior bulbar conjunctiva while other parts of the ocular surface (lid, cornea, and other regions of conjunctiva) could also be affected in AC.

In summary, our study characterizes and contextualizes the conjunctival microbiome dysbiosis in AC. The microbiome signatures identified here represent potential targets for follow-up studies on the microbial mech- 
anisms that underlie AC and other non-infectious ocular surface inflammations.

\section{ACKNOWLEDGMENTS}

This work was supported by the National Natural Science Foundation of China 81700803 and 81770892.

\section{CONFLICT OF INTEREST}

No potential conflict of interest relevant to this article was reported.

\section{AUTHOR CONTRIBUTIONS}

L.W. and L.L. conceived the study; Q.L., Yu L, and B.Z. performed data analysis; Jing L., S.Z., Yinglin L., and X.D. collected clinical samples; X.W., S.G., and Juanran L performed the metagenomic sequencing experiments; Q.L. and Jing L. drafted the manuscript.

\section{REFERENCES}

1. Pawankar R. Allergic diseases and asthma: a global public health concern and a call to action. World Allergy Organ J . 2014;7(1):12.

2. La Rosa M, Lionetti E, Reibaldi M, et al. Allergic conjunctivitis: a comprehensive review of the literature. Ital J Pediatr . 2013;39(1):18.

3. Kemter AM, Nagler CR. Influences on allergic mechanisms through gut, lung, and skin microbiome exposures. J Clin Invest . 2019;129(4):1483-1492.

4. Lunjani N, Satitsuksanoa P, Lukasik Z, Sokolowska M, Eiwegger T, O'Mahony L. Recent developments and highlights in mechanisms of allergic diseases: Microbiome. Allergy Eur J Allergy Clin Immunol . 2018;73(12):2314-2327.

5. Geoghegan JA, Irvine AD, Foster TJ. Staphylococcus aureus and Atopic Dermatitis: A Complex and Evolving Relationship. Trends Microbiol . 2018;26(6):484-497.

6. Teo SM, Mok D, Holt KE, et al. The Infant Nasopharyngeal Microbiome Impacts Severity of Lower Respiratory Infection and Risk of Resource The Infant Nasopharyngeal Microbiome Impacts Severity of Lower Respiratory Infection and Risk of Asthma Development. Cell Host Microbe . 2015;17(5):704-715.

7. Dong Q, Brulc JM, Iovieno A, et al. Diversity of bacteria at healthy human conjunctiva. Investig Ophthalmol Vis Sci . 2011;52(8):5408-5413.

8. Shivaji S, Jayasudha R, Prashanthi GS, Chakravarthy SK, Sharma S. The human ocular surface fungal microbiome. Investig Ophthalmol Vis Sci . 2019;60(1):451-459.

9. Doan T, Akileswaran L, Andersen D, et al. Paucibacterial microbiome and resident DNA virome of the healthy conjunctiva. Investig Ophthalmol Vis Sci . 2016;57(13):5116-5126.

10. Wen X, Miao L, Deng Y, et al. The Influence of Age and Sex on Ocular Surface Microbiota in Healthy Adults. Investig Opthalmology Vis Sci . 2017;58(14):6030.

11. Takamura E, Uchio E, Ebihara N, Ohno S, Ohashi Y. Allergology International Japanese guidelines for allergic conjunctival diseases 2017 *. Allergol Int . 2017;66(2):220-229.

12. Deng Y, Wen X, Hu X, et al. Geographic Difference Shaped Human Ocular Surface Metagenome of Young Han Chinese From Beijing, Wenzhou, and Guangzhou Cities. Investig Opthalmology Vis Sci . 2020;61(2):47.

13. Bolger AM, Lohse M, Usadel B. Trimmomatic: A flexible trimmer for Illumina sequence data. Bioinformatics . 2014;30(15):2114-2120.

14. Schmieder R, Edwards R. Quality control and preprocessing of metagenomic datasets. Bioinformatics . 2011;27(6):863-864. 
15. Wood DE, Lu J, Langmead B. Improved metagenomic analysis with Kraken 2. Genome Biol . $2019 ; 20(1): 257$.

16. Franzosa EA, McIver LJ, Rahnavard G, et al. Species-level functional profiling of metagenomes and metatranscriptomes. Nat Methods . 2018;15(11):962-968.

17. Alcock BP, Raphenya AR, Lau TTY, et al. CARD 2020: Antibiotic resistome surveillance with the comprehensive antibiotic resistance database. Nucleic Acids Res . 2020;48(D1):D517-D525.

18. Buchfink B, Xie C, Huson DH. Fast and sensitive protein alignment using DIAMOND. Nat Methods . 2014;12(1):59-60.

19. Davis NM, Proctor DiM, Holmes SP, Relman DA, Callahan BJ. Simple statistical identification and removal of contaminant sequences in marker-gene and metagenomics data. Microbiome . 2018;6(1):226.

20. Jervis-Bardy J, Leong LEX, Marri S, et al. Deriving accurate microbiota profiles from human samples with low bacterial content through post-sequencing processing of Illumina MiSeq data.Microbiome . 2015;3(1):19.

21. Segata N, Izard J, Waldron L, et al. Metagenomic biomarker discovery and explanation. Genome Biol . 2011;12(6):R60.

22. Ablashi D., Berneman Z., Kramarsky B, Whitman J, Asano Y, Pearson G. Human herpesvirus-7 (HHV7): current status. Clin Diagn Virol . 1995;4(1):1-13.

23. Brodská P, Panzner P, Pizinger K, Schmid-Grendelmeier P. IgE-mediated sensitization to malassezia in atopic dermatitis: More common in male patients and in head and neck type. Dermatitis . 2014;25(3):120-126.

24. Glatz M, Bosshard PP, Hoetzenecker W, Schmid-Grendelmeier P. The Role of Malassezia spp. in Atopic Dermatitis. J Clin Med . 2015;4(6):1217-1228.

25. Shin H, Price K, Albert L, Dodick J, Park L, Dominguez-Belloa MG. Changes in the eye microbiota associated with contact lens wearing. MBio . 2016;7(2):1-6.

26. Solomon A. Allergic manifestations of contact lens wearing.Curr Opin Allergy Clin Immunol . 2016;16(5):492-497.

27. Alnahas S, Hagner S, Raifer H, et al. IL-17 and TNF- $\alpha$ Are Key Mediators of Moraxella catarrhalis Triggered Exacerbation of Allergic Airway Inflammation. Front Immunol . 2017;8(NOV):1-11.

28. Rahman Fink N, Chawes BL, Thorsen J, et al. Neonates colonized with pathogenic bacteria in the airways have a low-grade systemic inflammation. Allergy . 2018;73(11):2150-2159.

29. Pascal M, Perez-Gordo M, Caballero T, et al. Microbiome and Allergic Diseases. Front Immunol . $2018 ; 9$ (JUL).

30. Bonini S, Coassin M, Aronni S, Lambiase A. Vernal keratoconjunctivitis. Eye . 2004;18(4):345-351.

31. Goleva E, Jackson LP, Harris JK, et al. The effects of airway microbiome on corticosteroid responsiveness in asthma. Am J Respir Crit Care Med . 2013;188(10):1193-1201.

32. Shimizu E, Ogawa Y, Saijo Y, et al. Commensal microflora in human conjunctiva; characteristics of microflora in the patients with chronic ocular graft-versus-host disease. Ocul Surf . 2019;17(2):265-271.

33. Lloyd-Price J, Mahurkar A, Rahnavard G, et al. Strains, functions and dynamics in the expanded Human Microbiome Project. Nature . 2017;550(7674):61-66.

FIGURE LEGENDS 
FIGURE 1. Conjunctival microbiome compositions of healthy and AC participants. A, Microbiome of healthy subjects $(n=48)$ and AC patients with SAC/PAC $(n=21)$ or VKC $(n=18)$ classified at the kingdom level. B, Microbiome of healthy subjects and AC patients classified at the genus level.

FIGURE 2 . Taxonomic differences between individuals with AC $(n=39)$ and healthy individuals $(n=48)$. A, Alpha diversity measured with the Shannon index was computed for AC and healthy samples. B, Beta diversity measured with Bray-Curtis dissimilarity within AC and healthy samples. $P$ values of alpha and beta diversity were computed using a two-sided Wilcoxon test. C, PCoA of samples from all 87 participants based on species-level Bray-Curtis distance. D, LDA effect sizes of species differentially abundant (LDA effect size $>4$ ) in the participants with and without AC. E, Relative abundance distributions of species enriched and reduced in AC. Species with fold change $>5$ were displayed. Relative abundances in the heatmap were centered and scaled across all the samples.

FIGURE 3 . Functional differences between individuals with AC $(n=39)$ and healthy individuals $(n=48)$. A, KEGG orthology, EC gene families, EggNOG clusters of orthologous groups, and Pfam protein families that are enriched in AC patients versus healthy subjects. Only the protein family signatures identified with LEfSe (Kruskal-Wallis rank-sum test $P<0.05$ and LDA effect size $>2$ ) are displayed in the scatter plots. The gene families (enriched in AC) with LDA effect size $>3$ are highlighted in red. RP, ribosomal protein. $\mathrm{B}$, Prevalence of CARD antibiotic resistance genes in the conjunctival metagenome from patients with AC and healthy individuals.

FIGURE 4 . Microbiome differences between individuals with SAC/PAC and VKC. A, Alpha diversity measured with the Shannon index was computed for healthy subjects $(n=48)$ and patients with SAC/PAC $(n=21)$ and VKC $(n=18)$. B, Beta diversity measured with Bray-Curtis dissimilarity within healthy, $\mathrm{SAC} / \mathrm{PAC}$, and VKC samples. C, PCoA of samples from $39 \mathrm{AC}$ participants with SAC/PAC or VKC based on species-level Bray-Curtis distance. D, LDA effect sizes of species differentially abundant (LDA effect size $>3)$ in $\mathrm{SAC} / \mathrm{PAC}$ or VKC.

FIGURE 5 . Relative abundance distributions of species differentially abundant between individuals with SAC/PAC and VKC. A, Overview of the distributions of species associated with the two types of AC. Relative abundances in the heatmap were centered and scaled across the samples from individuals with AC. B, Relative abundances of the species enriched in SAC/PAC. C, Relative abundances of the species enriched in VKC. Only the species that were present in $>50 \%$ samples with mean relative abundances $>0.1 \%$ in the overabundant group were displayed in the B and C.

\section{FIGURE 1}



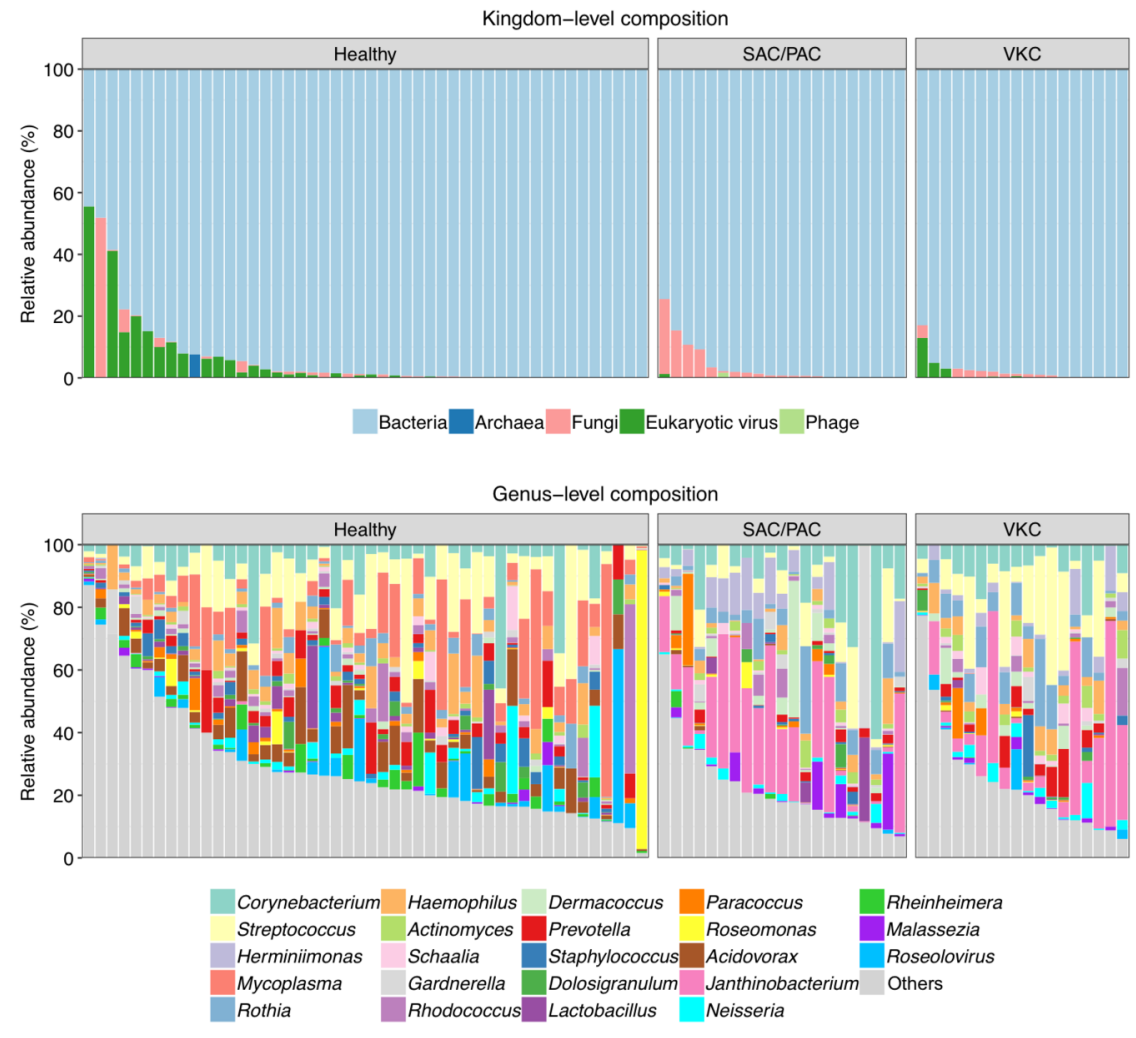

(A)

(B)

FIGURE 2 

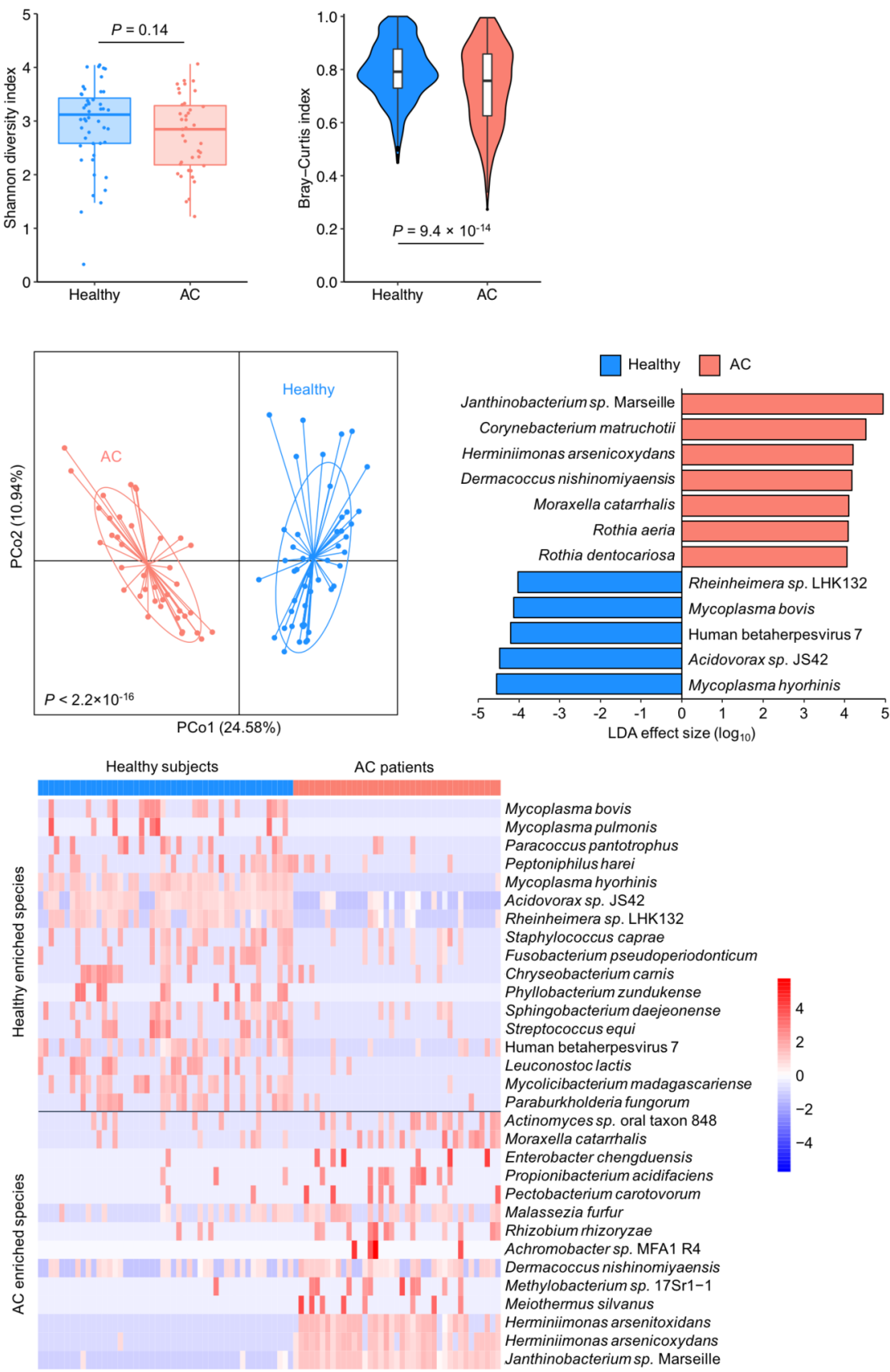
(A)
(B)
(C) (D)
(E)

FIGURE 3 

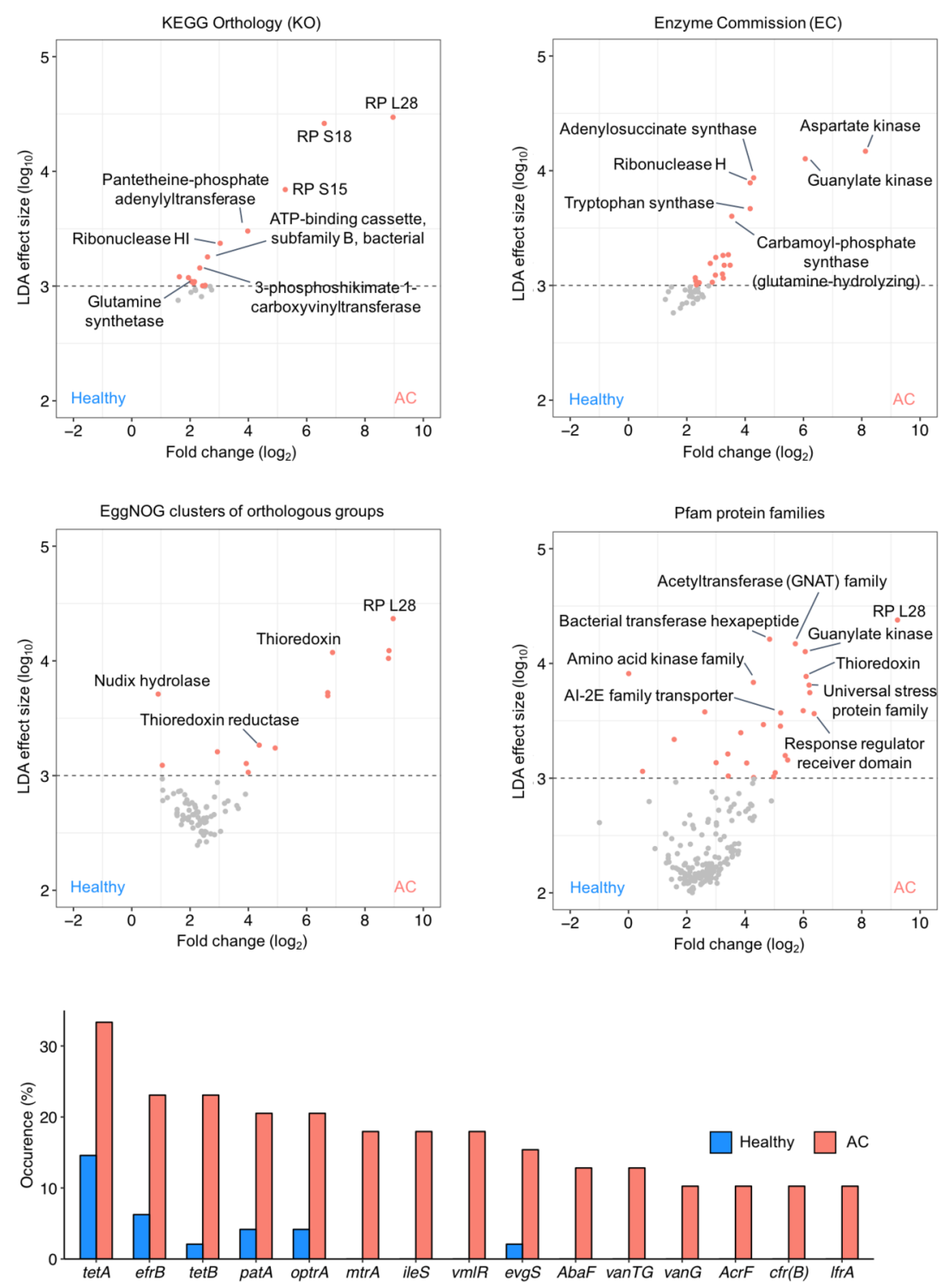

(A)

(B) 

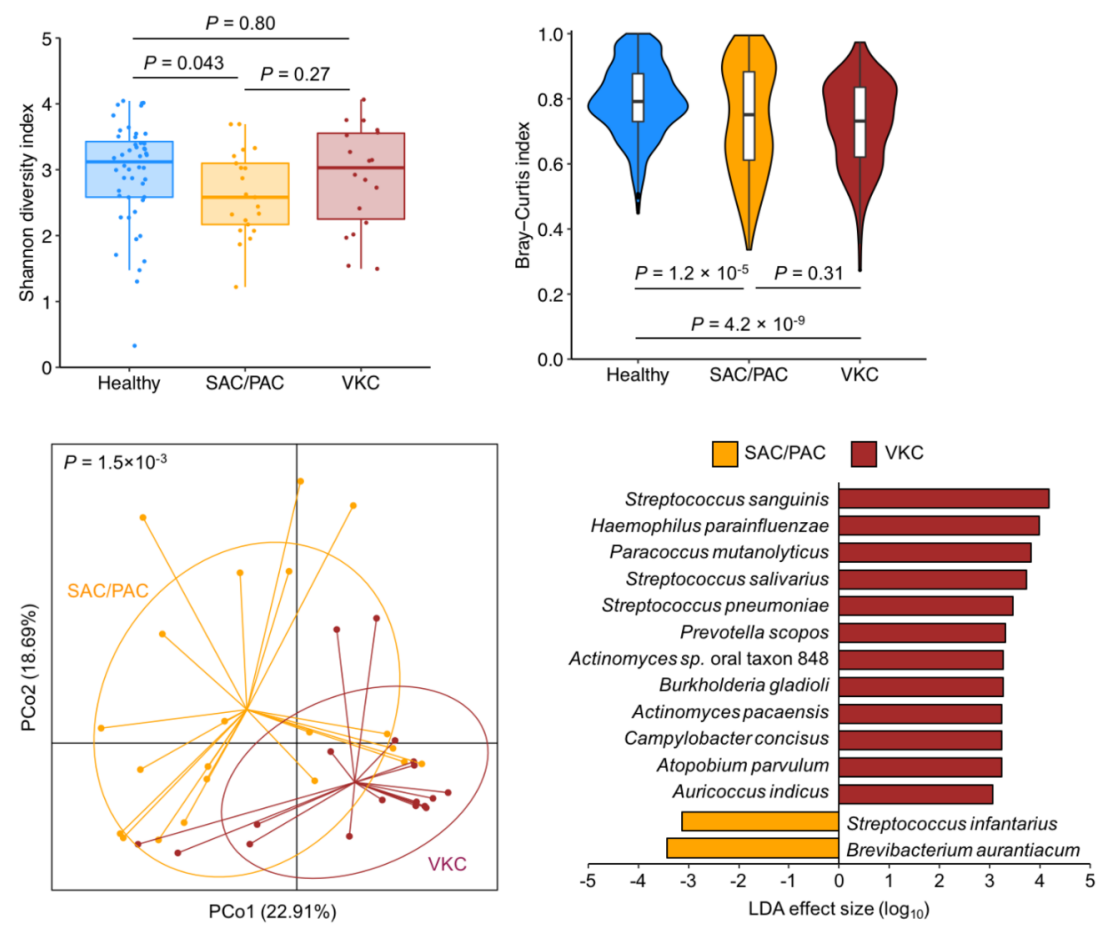

FIGURE 4

(A) (B)

(C) (D) 

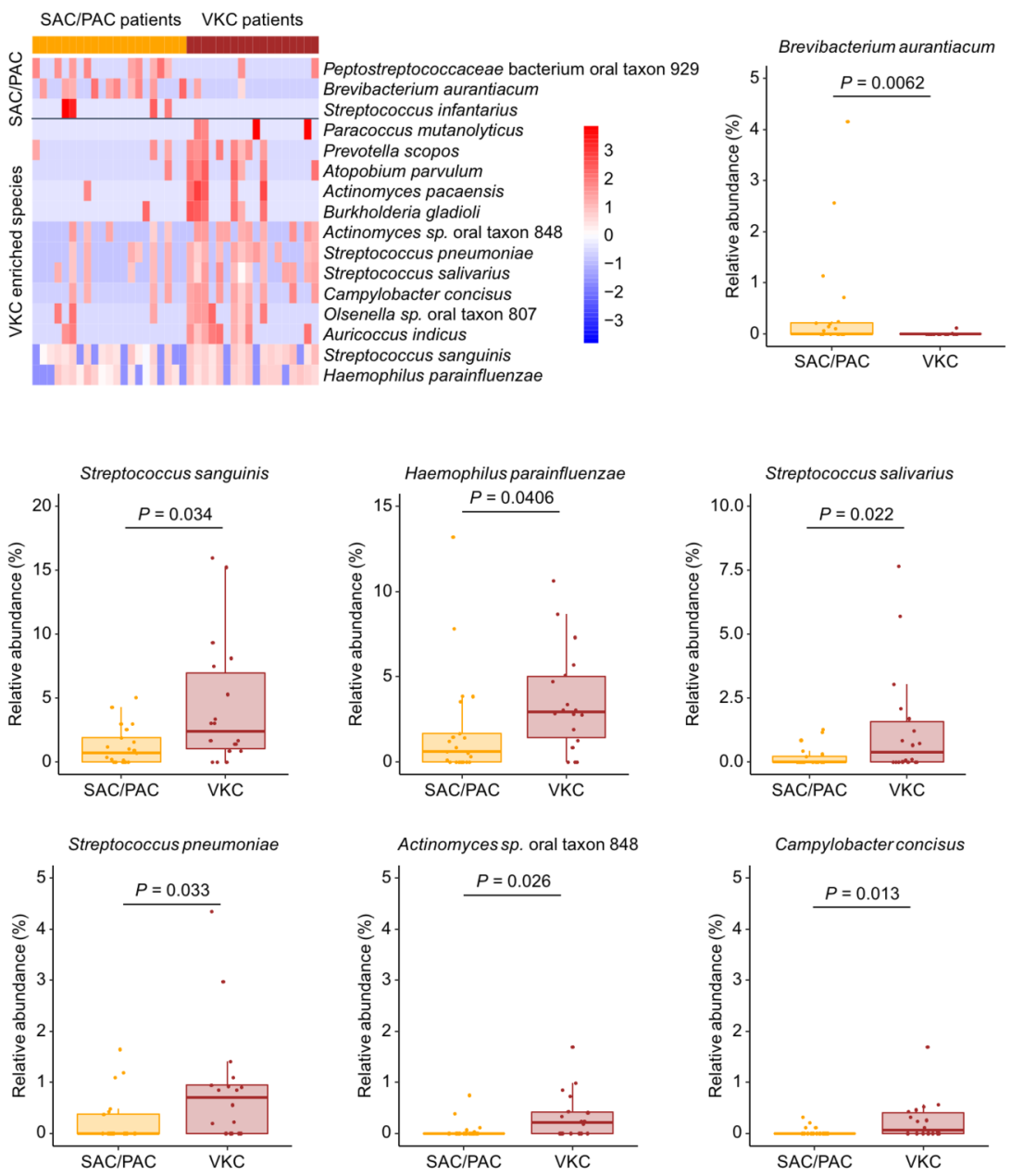

\section{FIGURE 5}

(A) (B)

(C)

TABLE S1 . Demographic Information of the healthy and AC participants

\begin{tabular}{llll}
\hline & Healthy & AC & $P$ value \\
\hline Number of samples & 48 & 39 & \\
Average age (Mean \pm SD) & $27.9 \pm 4.0$ & $19.8 \pm 16.7$ & 0.005 \\
Sex (Male/female) & $23 / 25$ & $25 / 14$ & 0.196 \\
\hline
\end{tabular}

Note: Student's t-Test for age and Chi-squared Test for sex. AC, Allergic conjunctivitis.

TABLE S2 . Demographic Information of the participants with SAC/PAC and VKC 


\begin{tabular}{llll}
\hline & SAC/PAC & VKC & $P$ value \\
\hline Number of samples & 21 & 18 & \\
Diagnosis & 7 SAC, 14 PAC & 18 VKC & \\
Average age (Mean \pm SD) & $22.3 \pm 19.5$ & $17.0 \pm 12.6$ & 0.316 \\
Sex (Male/female) & $11 / 10$ & $14 / 4$ & 0.189 \\
\hline
\end{tabular}

Note: Student's t-Test for age and Chi-squared Test for sex. SAC, seasonal allergic conjunctivitis; PAC, perennial allergic conjunctivitis; VKC, vernal keratoconjunctivitis. 